\title{
Mammalian Phospholipid Homeostasis: Homeoviscous Adaptation Deconstructed by Lipidomic Data Driven Modelling
}

Marcus K. Dymond* ${ }^{1}$

*Author for correspondence; M.Dymond@brighton.ac.uk

${ }^{1}$ Division of Chemistry, School of Pharmacy and Biological Sciences, University of Brighton, BN2 4GL.

\section{Keywords}

Membrane Order; Spontaneous Curvature; Homeoviscous Adaptation; Intrinsic Curvature Hypothesis; Phospholipid Homeostasis

\section{Abstract}

One of the mostly widely cited theories of phospholipid homeostasis is the theory of homeoviscous adaptation (HVA). HVA states that cells maintain membrane order (frequently discussed in terms of membrane fluidity or viscosity) within tight conditions in response to environmental induced changes in membrane lipid composition. In this article we use data driven modelling to investigate membrane order, using methodology we previously developed to investigate another theory of phospholipid homeostasis, the intrinsic curvature hypothesis. A set of coarse-grain parameters emerge from our model which can be used to deconstruct the relative contribution of each component membrane phospholipid to net membrane order. Our results suggest, for the membranes in the mammalian cells we have studied, that a ratio control function can be used to model membrane order. Using asynchronous cell lines we quantify the relative contribution of around 130 lipid species to net membrane order, finding that around 16 of these phospholipid species have the greatest effect in vivo. Then using lipidomic data obtained from partially synchronised cultures of HeLa cells we are able to demonstrate that these same 16 lipid species drive the changes in membrane order observed around the cell cycle. Our findings in this study suggest, when compared with our previous work, that cells maintain both membrane order and membrane intrinsic curvature within tight conditions. 


\section{Introduction}

The theory of homeoviscous adaptation (HVA) builds on long-standing observations that the phospholipid composition of many organisms adjusts in response to changes in environmental temperature (Hazel, 1995). In development of HVA, Sinensky (Sinensky, 1974) observed using Electron Spin Resonance (ESR) that the order parameter of E. coli membranes remained constant, determined as a function of spin probe rotational correlation time, despite their phospholipid compositions changing in response to different growth temperatures. Subsequently, evidence for HVA as a conserved evolutionary mechanism emerged (Behan-Martin et al., 1993; Cossins and Prosser, 1978) and HVA has since been reported under many other changing environmental conditions, such as in response to hydrostatic pressure (Behan et al., 1992; Cossins and Macdonald, 1986, 1984), osmotic pressure (Laroche et al., 2001) , low magnetic field strength (Santoro et al., 1997) and chemicals such as PCB-153 (Gonzalez et al., 2013), mitelfosine (Rybczynska et al., 2001) and crude oil (Mazzella et al., 2005). To date evidence for HVA has been found in species across the three domains of life and it is arguably the most widely used theory to explain phospholipid compositional changes, usually through the concept of maintaining membrane fluidity or membrane viscosity. Further examples of HVA, in addition to those given above are in mammalian cell culture lines (Anderson et al., 1981), zooplankton (Gladyshev et al., 2011), Antarctic bacteria (Chattopadhyay and Jagannadham, 2001) and soy bean seedlings (Davy De Virville et al., 2002) as reviewed (Hazel, 1997, 1995; Vigh et al., 1998). It should be noted however that, whilst the terms membrane fluidity and membrane viscosity are in common usage, their physics arise from 3-dimensional molecular translation in liquids and, as such, these terms are not suited to describe translation in 2-dimensional membranes (Gawrisch, 2005). Thus it is preferable to discuss HVA in terms of preservation of membrane order rather than membrane 'viscosity' or membrane 'fluidity'.

Mechanistic insights into HVA have implicated the ratio of saturated to unsaturated fatty acids (ratio sats/unsats) as a critical membrane order modulation pathway in vivo. The most compelling evidence for this mechanism stems from data where the ratio sats/unsats follows a linear progression when plotted against the membrane fluorescence polarisation of diphenyl hexatrine (DPH) (Cossins and Prosser, 1978), for animal species adapted to different mean habitation temperatures, as reviewed (Hazel, 1995). The argument that 
underpins the observation that the ratio sats/unsats modulates membrane order arises from the gel $\left(\mathrm{L}_{\beta}\right)$ to fluid lamellar $\left(\mathrm{L}_{\alpha}\right)$ phase transition temperature $\left(T_{m}\right)$ values (Koynova and Caffrey, 1998) of the saturated and unsaturated lipid membrane components. Above the $T_{m}$ the chains of phospholipids are disordered, below it they are ordered, with a gradual increase in order observed as the $T_{m}$ is approached from above (Morrow et al., 1992). As a general rule across a homologous headgroup series, increases in saturated hydrocarbon chain length increase the value of $T_{m}$ and increasing cis unsaturation decreases the value of $T_{m}$. The position of the cis unsaturation and branching in the chain has an effect on the $T_{m}$ value as does the individual headgroup i.e. phosphatidylethanolamine (PE) or phosphatidylcholine (PC) (Cevc, 1991; Marsh, 2013, 1999), however the introduction of a single unsaturation into a saturated chain causes one of the biggest changes to $T_{m}$ and membrane order. Thus the ratio sats/unsats accounts for the major structural contribution of each individual compositional lipid species to the mixed membrane order parameter. Several other metrics have also been shown to correlate broadly with in vivo membrane order, these are the PC: PE ratio and the unsaturation index (i.e. the sum of each fatty acid concentration multiplied by the number of its unsaturations) (Anderson et al., 1981; Bernabé Bloj et al., 1973). Ideally membrane order parameters, rather than $T_{m}$ values, of the individual component lipids should be used to rationalise their effect on the average membrane order, however the literature does not contain enough of these order parameters to enable this for the complex mix of lipids in biological membranes. Hence qualitatively, within the HVA community, the effects of individual lipid compositional changes on membrane order are estimated from a phospholipid species' $T_{m}$ value. Such that at a fixed environmental temperature, the difference (in temperature units) of the individual lipids from their $T_{m}$, weighted by their composition, represents a crude method for estimating the order they impart on a homogenous bilayer system. One of the limitations of this methodology is that the membrane order of individual lipids is often dependent on the other lipids in the system. For example in model membranes cholesterol increases the membrane order of phospholipid membranes as reported (Kaiser et al., 2009).

Within the environmental context of HVA theory decreases in temperature, which will drive an increase in membrane order, are thought to be mediated homeostatically through the elevation of unsaturated lipid levels, which decrease membrane order. Of 
course since the different lipid headgroups also have different contributions to the average membrane order there are a range of other lipid substitution mechanisms through which HVA might exhibit. These mechanisms are discussed in depth for Archaea by Oger and Cairo (Oger and Cario, 2013) and poikilotherms by Guschina and Harwood (Guschina and Harwood, 2006). Observations that cholesterol (Crockett and Hazel, 1995) has a regulatory role in preserving membrane order demonstrate that the modification of phospholipid composition is not the only mechanism through which membrane order can be regulated. Equally, however, observations that cholesterol (King and Spector, 1978) content is unchanged despite significant changes in fatty acid composition in Ehrlich ascites suggests cholesterol is not ubiquitous in regulating membrane order. These potential contradictions illustrate that a broad one-fit model to explain the preservation of membrane order in vivo is unlikely. Instead it seems probable that different membranes adapted for different functions may use different mechanisms to restore membrane order (Crockett and Hazel, 1995). Additionally, work over the last decade has suggested that, rather than being a homogenous bilayer mixture, cell membranes are heterogeneous and contain microdomains of cholesterol, sphingomyelin, proteins and to a lesser extent saturated lipids (Simons and Sampaio, 2011). These 'lipid rafts' are widely accepted to be analogous to the disordered $\left(L_{d}\right)$ and ordered $\left(L_{o}\right)$ lipid phases observed in binary and tertiary lipid mixtures containing cholesterol (Kaiser et al., 2009). A number of other experimental factors further complicate the interpretation of HVA phenomena. Firstly the membrane order parameter is segmental, with respect to the length of phospholipid chains. And secondly, different membrane fluorescence probes sit at different depths in the membrane. In the past, failure to recognise the latter has caused conflicting data in the literature, as discussed (Demchenko et al., 2009).

One anomaly in HVA theory is that many species exposed to colder temperatures accumulate both polyunsaturated fatty acids (Cossins and Macdonald, 1986; Hazel, 1995) and monounsaturated fatty acids. This is tricky to reconcile with any mechanism whereby membrane order is the only property being maintained, since membrane order can be just as easily restored by accumulating monounsaturated fatty acids (Hazel, 1995). The theory of homeophasic adaptation (Vigh et al., 1998) suggests that control mechanisms might be in operation to maintain phospholipid membrane composition away from the inverse 
hexagonal phase transition. Other theories of phospholipid homeostasis (Hermansson et al., 2011), such as the intrinsic curvature hypothesis (Gruner, 1985) have emerged in response to these observations. It has also been suggested that cholesterol homeostasis enables cells to control lateral phase separation of their membranes such that lipid raft size and aggregation is controlled (Simons and Ikonen, 2000). In previous work (Dymond et al., 2008, 2013; Dymond and Attard, 2008; Hague et al., 2013) we have presented detailed explanations of the intrinsic curvature hypothesis, which maintains that cells regulate membrane curvature elastic stress throughout phospholipid homeostasis. Both HVA and the intrinsic curvature hypothesis are post-translational theories of phospholipid homeostasis. At the transcriptional level, membrane free fatty acids are implicated in the mechanisms of phospholipid homeostasis. The peroxisome proliferator-activated receptors (PPARs) are nuclear receptors that act as transcription factors, sensing fatty acids and lipid metabolites (Varga et al., 2011). Similarly the sterol-regulatory element binding proteins (SREBPs) activate the expression of around 30 genes involved in the synthesis and uptake of fatty acids, cholesterol and phospholipids (Georgiadi and Kersten, 2012). In E.coli the FadR transcription factor binds to DNA mediated by long chain acyl-CoAs (Xu et al., 2001), forming part of a pathway through which saturated and unsaturated membrane fatty acid levels control the expression of proteins involved in phospholipid homeostasis (Parsons and Rock, 2013). Disentangling the different transcriptional and post-translational control mechanisms within phospholipid homeostasis is an ongoing challenge that requires new analytical tools suited to systematically analyse the large number of molecular species that make up omic data sets.

\subsection{Mechanistic insights into HVA from lipid compositional data}

The strength of the correlation between membrane order and lipid composition has led to several metrics of HVA, such as the ratio sats/unsats, the unsaturation index or the PC/ PE ratio being used to infer changes in cellular membrane fluidity (Anderson et al., 1981; Bernabé Bloj et al., 1973; Calder et al., 1990; Cooper et al., 2014; Ozawa, 2011; Yu et al., 2014) and the mechanisms by which such changes occur. It should be noted that as early as 1986, Cossins and Macdonald (Cossins and Macdonald, 1986) demonstrated that the ratio 
sats/ unsats and the unsaturation index are not a perfect metric for HVA, concluding that it is likely that these measures do not take account of many of the subtleties of the relationship between lipid composition and membrane order.

One disadvantage of the ratio sats/unsats is that it is derived from free fatty acids (or molecular derivatives thereof) and thus its production typically requires chemical treatment to render all component lipids into their fatty acid composites, which are then quantified by HPLC. This approach results in the loss of a significant amount of lipid structural detail. Progress in the field of lipidomics means that quantitative phospholipid compositions can now be relatively easily obtained with a higher level of structural confidence. This presents an opportunity to generate evidence based (data driven) models for membrane properties, which emerge from the net contributions of a large number of individual components, a method that in principle allows the physics of biological membranes to be reconstructed within a systematic framework. It should however be noted that a number of experimental limitations currently necessitate a coarse-grained deconstruction of the properties of biological membranes. These arise from the limitation of lipidomics to provide quantitative structural information of the many isomeric phospholipid species that occur in cells.

\subsection{Data driven modelling of control functions using quantitative lipidomic datasets}

Recently we reported evidence for a homeostatic control mechanism in the phospholipid biosynthetic network of immortalised human cell lines. Our findings were the result of a tandem computational and experimental strategy using data driven modelling in combination with shotgun lipidomics (Dymond et al., 2013). The control mechanism we sought evidence for was a ratio control (Wade, 2004) mechanism derived from the intrinsic curvature hypothesis (Gruner, 1985), which we found was conserved with a coefficient of variance of $10 \%$ across 45 cell populations from three different cell lines, each with a unique phospholipid compositional profile.

We now use the same techniques and the same datasets to search for correlations using a control function parameterised for membrane order. Ideally, the order parameters of the individual component lipids in pure systems, at fixed bilayer depth, ought to be used 
to rank the contribution of individual lipids to mixed membrane order. However, currently the published data density of lipid order parameters is too low to enable this for each of lipids found in biological membranes, thus we use in vitro $T_{m}$ values to rank the relative contributions of the component lipids to membrane order. Our ratio control equation is formulated as below;

$$
p_{\text {dis }}=\frac{\sum_{n=0}^{b} w_{n}\left[L_{d i s, n}\right]}{\sum_{m=0}^{a} \frac{1}{w_{m}}\left[L_{\text {ord }, m}\right]} \quad \text { Equation 1, }
$$

which addresses the following question: given the set of all possible lipid species $\{L\}$, is there a universal pivot species, $L_{p}$, that defines how the set can be partitioned into subsets $\left\{L_{\text {ord }}+L_{p}\right\},\left\{L_{\text {dis }}\right\}$ such that the mean average ratio control function for membrane disorder $p_{\text {dis, }}$ has the minimum variance across the range of our independent lipidomic data sets?

In Equation 1 [ $L_{d i s, n}$ ] denotes the concentration of the disordering lipid $n$ and $w_{n}$ is a weighting factor for lipid $n,\left[L_{o r d, m}\right]$ is the concentration of the ordering lipid $m$ and $w_{m}$ is the weighting factor for lipid $m$. Lipids with $w$ values below that of the pivot lipid $\left(L_{p}\right)$ will increase membrane disorder and lipids with $w$ values above that of the pivot will increase membrane order. The variables $a$ and $b$ are the total numbers of ordering and disordering lipids respectively. Values of $w_{n}$ and $w_{m}$ are generated by a priori ranking of the structural contributions of each lipid to membrane order, guided by in vitro trends in individual lipid $T_{m}$ values, as discussed in the methods section. Higher values of $p_{\text {dis }}$ equate with more disordered hydrocarbon chains.

Using the algorithm and quantitative lipidomic data for the HeLa, HL60 and HL60 oleate cells (HL60 cells cultured with exogenous oleate) we have previously presented (Dymond et al., 2013), we separate the data into training and test sets. Then using randomly generated parameter sets to obtain values of $w$, see methods section, we calculate the mean $p_{\text {dis }}$ and the standard deviation for the test lipidome $(\mathrm{HL60}$ oleate $), p_{\text {dis oleate. }}$. We then calculate the coefficient of variance ( $\left.c_{v \text { oleate }}\right)$ of $p_{\text {dis oleate }}$ and repeat through $1 \times 10^{4}$ iterations, using each lipid species in the lipidome as the pivot lipid. This gives us an extensive number of test parameter sets that can be used to construct values of $w$ for each component lipid in the training lipidomes. For each of these test parameter sets, using the same control function in conjunction with the lipidomic data from the training sets (i.e. the 
HeLa and HL60 lipidomes), the mean average $p_{\text {dis HL6O }}$ and $p_{\text {dis HeLa }}$, associated standard deviations and $c_{v H L 6 O}$ and $c_{v \text { HeLa }}$ are calculated Finally, we calculate the mean coefficient of variance $c_{v \text { mean }}$ i.e. the mean average $c_{V \text { oleate, }} c_{V H L 60}$ and $c_{V \text { HeLa }}$ and the standard deviation of $c_{v \text { mean }}$. The lower the value of the $c_{v \text { mean }}$ the stronger the evidence for the existence of the control function in the test and training lipidomes. As a rule of thumb $c_{v \text { mean }}$ values of $10 \%$ and lower we consider to be strongly evidenced, in the range of $10 \%$ to $20 \%$ we consider weakly evidenced and above $20 \%$ we consider irrelevant. This process is shown schematically in Figure S1.

One advantage of this data driven approach is that the solutions that emerge are inherently linked to the large volume of experimental data and therefore low $c_{v}$ mean values indicate the validity of the model. Another advantage is that whilst the control function is constructed in a linear form, in the sense that the individual molefraction of the components ranked by their phase transition temperature, $T_{m}$, are used to generate a set of parameters, the random iterative nature through which $w$ is determined means the model can find non-linear relationships between the lipid composition and the membrane property of interest. It should however be noted that the parameters ( $w$ values) that emerge from these models represent the average contribution to membrane order of individual component lipids in the system. This is significant because the presence of other lipids like cholesterol can have an ordering effect on phospholipids, an effect that is most significant for saturated lipids (Pan et al., 2008).

The critical difference between this and our previous work is the a priori ranking of the contribution of each individual lipid to each control function. For the intrinsic curvature hypothesis the relative contribution of lipid headgroups to net stored elastic energy decrease in the order diacylglycerol (DAG) > phosphatidic acid (PA) > PE > phosphatidylinositol (PI) > PC > phosphatidylserine (PS), whilst the contribution to membrane disorder decreases in the order DAG $>$ PI $>$ PC $>$ PS $>$ PC $>$ PA, as justified in the Material and Methods section. In both theories membrane curvature elastic stress and membrane disorder increase with the number of unsaturations in a hydrocarbon chain, although it should be noted there is no expectation that this should occur proportionally. It should also be noted that lipidomic data used in this study are for the PC, PE, PS, PI, PA, DAG headgroups. Since these lipids are predominantly found within the proposed liquid 
disordered phase in biological membranes then, in the strictest sense; this study assesses membrane order in this phase.

In this publication we seek to provide insights into two questions; firstly, can we uncover evidence that membrane order modulation is occurring in parallel to intrinsic curvature modulation within the same data sets? And secondly, can our best parameter sets be used to systematically deconstruct the individual lipid contributions to membrane order seen in vivo? 


\section{Materials and Methods}

All lipidomic and cell culture experimental methods plus extensive details of the data driven modelling methodology have been published previously (Dymond et al., 2013; Hague et al., 2013). The lipidomic datasets used here are freely available in the supplementary information of the above publications. For the purposes of data driven modelling the three cell sets, HeLa, HL-60 and HL-60 oleate (the HL-60 cell line cultured with exogenous oleate) were used as test and training data sets. The HL-60 oleate data was the training set and the HL-60 and HeLa data were the test data sets 1 and 2 respectively.

\subsection{Ratio control functions formalised for membrane disorder}

To be consistent with our previous methodology (Dymond et al., 2013) we coarsegrained the individual contributions of the composite lipid species to net membrane order. Our coarse-graining of the phospholipid molecular structure takes account of the number of unsaturations per fatty acid ( 0 to 6 ) as well as different fatty acid and headgroup combinations. Chain length is not modelled for, thus we write all modelled phospholipid structures in the form of headgroup, number of unsaturations in first fatty acid and number of unsaturations in second fatty acid. Therefore PC 0:1 will refer to any PC lipid with a saturated and monounsaturated fatty acid combination regardless of chain length or position of unsaturation.

Values of $w_{n}$ and $w_{m}$ were constructed from Equation 2

$$
w=\left(c_{h 1}+c_{h 2}\right) H_{g} \quad \text { Equation } 2 .
$$

Where values of $c_{h 1}$ and $c_{h 2}$ are the net contribution of each fatty acid chain to $w$ and $H_{g}$ is the headgroup contribution. Values of $c_{h 1}$ and $c_{h 2}$ were selected, based on individual lipid structure, from a list of randomly generated numbers between 0.00 and 1.00 , ranked $a$ priori such that for each fatty acid unsaturation, within a lipid hydrocarbon chain, the disorder imparted on the membrane increases with $c_{h 1} 0<c_{h 1} 1<c_{h 1} 2<c_{h 1} 3<c_{h 1} 4<c_{h 1} 5<$ $c_{h 1} 6$, where the integer is the number of unsaturations in the fatty acid chain. Values of $c_{h 2}$ 
were similarly generated. This is in accordance with the trends in $T_{m}$ values seen in the literature, summarised in Table 1.

For example PC $18: 0 / 18: 0$ has a $T_{m}$ of $55.3^{\circ} \mathrm{C}$, which falls to $5.6{ }^{\circ} \mathrm{C}\left(\mathrm{PC} 18: 0 / 18: 1 \mathrm{c} \Delta^{9}\right)$ through the introduction of one unsaturation. Similar, but less pronounced drops are seen when successive hydrocarbon chain unsaturations are added. Similarly, $T_{m}$ values for each of the phospholipid headgroups were used to rank the individual headgroup $H_{g}$. These are summarised in Table 1 and ranked such that the contribution to disorder is $H_{g P A}<H_{g P E}<H_{g P S}$ $<H_{g P C}<H_{g P I}<H_{g D A G}$. In the particular case of DAG we could find no appropriate $T_{m}$ values for DAG that would allow ranking, however ESR studies show that dipalmitoylglycerol (DPG) decreases the membrane order parameter of dipalmitoyIPC vesicles (Ortiz et al., 1988). Similar studies point to the 'fluidising' ability of pure DAGs being slightly inferior to mixed chain PI (soy bean (Peng et al., 2012) and pig liver (Larijani and Dufourc, 2006)). Detailed profiles of the fatty acid distributions across the PI component lipids are unavailable but the reduced fatty acid profile of the soy PI (Avanti Polar Lipids) shows it is 31\% palmitoyl (16:0) and $50 \%$ linoleyl $\left(18: 2 c \Delta^{9,12}\right)$. As an upper limit, assuming the maximum concentration of PI 16:0/ 18:2 existed in the sample, then $19 \%$ of the $18: 2$ fatty acid would need to distributed across the remaining $7 \%$ stearyl (18:0), 5\% oleyl (18:1) and 7\% 18:3. Each of these potential PI combinations has a lower $T_{m}$ value than the PI 16:0/ 18:2c $\Delta^{9,12}$, suggesting all will elevate the disordering effect of PI 16:0/ 18:2c $\Delta^{9,12}$. In effect this means that the PI soy mixture is at most $60 \% \mathrm{PI} 16: 0 / 18: 2 c \Delta^{9,12}$, thus we conclude that pure DAG 16:0/ 18:2c $\Delta^{9,12}$ is likely to be more disordering of PC membranes than pure PI 16:0/ 18:2c $\Delta^{9,12}$, therefore we rank DAG after PI.

For each parameter set (generated iteratively) we calculate $p_{\text {dis }}$ for each cell population (flask) and then determine the arithmetic mean of $p_{\text {dis }}$ ( $p_{\text {dis oleate, }} p_{\text {dis HL6O }}$ and $p_{\text {dis }}$ HeLa) and standard deviation for each cellular lipidome ( $\mathrm{LLCO}_{\text {oleate, }} \mathrm{HL60}$ and HeLa). The coefficient of variance $c_{v}$ (i.e. the standard deviation/ mean expressed as a percentage) is calculated for each lipidome ( $c_{v \text { oleate, }} c_{v \text { HLGO }}$ and $\left.c_{v \text { HeLa }}\right)$ and the mean average $c_{v}\left(c_{v \text { mean }}\right)$ and standard deviation of the $c_{v}$ mean are computed and plotted against each other. Parameter 
sets with lowest variance across the training and test data sets appear towards the bottom left of these plots.

\section{Results and Discussion}

\subsection{Membrane disorder proxies from data driven modelling}

Using our lipidomic datasets we applied our data driven modelling techniques to look for parameter sets consistent with the preservation of membrane order using the methodology previously described (Dymond et al., 2013). Figure 1 shows the results of the data driven modelling, which are plots of the $c_{v \text { mean }}$ versus the standard deviation of the $c_{v}$ mean. Values to the lower left hand corner of these plots indicate parameter sets with the least variance across all (circa 45) of the cellular populations.

Figure 1 shows that the $c_{v \text { mean, }}$ for the different parameter sets tested against the control function for membrane order in Equation 1, have a variance as low as 6 to $8 \%$ and a standard deviation in the range of 0 to $5 \%$. Since we have previously reported, for a separate control function derived from the intrinsic curvature hypothesis, a $c_{v \text { mean }}$ of $10 \%$ and standard deviation close to zero (Dymond et al., 2013) the evidence for the existence of the HVA inspired control function is, superficially at least, comparable to the evidence for intrinsic curvature control function. The immediate implication of this result is that the preservation of membrane order is as well evidenced within our phospholipid data as membrane curvature elastic stress, since a comparable result has been arrived at through an identical process but with a different control function. This finding is consistent with the idea that the compositional changes we have reported in phospholipid membranes, across these three mammalian lipidomes (Dymond et al., 2013; Hague et al., 2013), occur to conserve both membrane order and intrinsic curvature (curvature elastic stress). To the best of our knowledge this is the first study to demonstrate any evidence of concomitant preservation of more than one membrane property across a set of in vivo lipid compositional changes. Before we discuss the implications of these findings, we consider how well these parameter sets capture the underlying trends of the individual lipid 
contributions to membrane order, choosing one parameter set to follow through and further analyse the data.

\subsection{Refining proxies of membrane disorder}

In principle any of the parameter sets in Table 2 could be used to deconstruct the individual lipid contributions to membrane disorder in any of the cell sets. However it is useful to consider if any of these parameter sets is individually better suited than the others to deconstruct the contribution of each lipid to membrane order. We briefly consider a number of different ways to overcome this problem however prior to this it is worth considering the limitations of our lipidomic data by way of guiding the decision.

As noted previously, we have measured the cellular concentration of PC, PE, PS, PA, $\mathrm{PI}$ and DAG lipids and used these to construct an evidence based control function, strictly speaking the data driven modelling is therefore only assessing the contribution of these lipids to net membrane order. Given all the evidence from model membranes, we would expect a contribution to membrane order from the lipid membrane components we have not measured, such as, the protein, cholesterol and sphingomyelin. However since the phospholipids we have quantified in our datasets are predominantly associated with the proposed liquid disordered phase, and other lipids like sphingomyelin are associated with the cholesterol-rich ordered domains (Kaiser et al., 2009) the parameter sets in Table 1 could be considered a proxy measure of the liquid disordered phases regions of the cell bilayer (Wu et al., 2013). In this instance the concentration of cholesterol and sphingolipids would be much lower than that observed in whole cell extracts and thus the membrane order induced by cholesterol would not be as significant.

Another point to consider is that, given the dynamic exchange of lipids laterally across lipid bilayers and between regions of different order in the membrane it is likely that single molecules of same lipid species will exist in different molecular environments such that each molecule might have different contributions to membrane order, depending on its neighbouring molecules. Hence the $w$ values that emerge from these studies effectively represent the average contribution of each lipid species to net membrane order. Bearing 
these considerations in mind, there are two ways to refine the data. Firstly, membrane order parameters derived from mixtures of phospholipids and cholesterol, below the composition of lateral phase separation into the $L_{d}$ and $L_{o}$ phases, could be plotted against their respective lipid values of $w$ generated from each of the parameter sets in Table 2. Secondly, the order parameters of pure lipids could be plotted against the respective lipid values of $w$ generated for each of the parameter sets in Table 2. In both cases the magnitude of $R^{2}$ could be used to select the best data set. This second method effectively suggests that all cholesterol exists in separate domains within the bilayer where it has little or no effect on the membrane order of the phospholipids present in the liquid disordered phase. However, experimental evidence in model membranes suggests that cholesterol has different solubility in (Wassall et al., 2004), and different ordering effects on, the acyl chains of (Pan et al., 2008) saturated, unsaturated and polyunsaturated lipid membranes. Thus the first method of refinement is preferable, the difficulty being that the concentration of cholesterol in the liquid disordered phase of complex lipid mixtures in vivo is not known. If it emerges that cholesterol is not abundant in this liquid disordered region then the average nature of the value of $w$, would suggest the second method is better suited, since it does not overestimate the role of cholesterol.

A search of the scientific literature looking for order parameter data that would enable refinement of the parameter sets by either of the methods above was made. To achieve our goal, order parameters for a series of phospholipids with the same headgroup and chain length and at least 0, 1, 2, 3, 4 unsaturations are required. Studies would also need to be performed at the same temperature and either with or without cholesterol at defined compositions. Additionally the order parameter of lipids with the PC, PE, PA, PI, PS, DAG headgroups with a fixed chain length and saturation both in the presence and absence of cholesterol would also be useful. Unfortunately we could find no studies in the literature that satisfied all these requirements individually or in a combination that allowed direct comparison; therefore as a next best option we referred back to the $T_{m}$ values shown in Table 1 to guide our selection.

As noted in section 1, within the historical context of HVA the gel to fluid lamellar transition temperatures of phospholipids has been used to qualitatively predict their effect on membrane order within biological membranes. In the absence of enough membrane 
order parameter data we plotted the $T_{m}$ temperatures shown in Table 1 for phospholipids with the isomeric fatty acid combinations found in mammalian cells i.e. the omega 3, 6 or 9 isomers against each of the values of $w$ that emerge from the parameter sets in Table 2. Using this method, plots of $T_{m}$ versus $w$ should tend towards a linear dependence such that the $R^{2}$ value could be used to refine the parameters in Table 2 . The limitation of this method is that $T_{m}$ is only a rough metric of membrane order in the pure lipid system. Plus this approach only considers pure lipid systems and takes no account of the ordering effect of lipids like cholesterol, which are greatest for saturated lipids. On the other hand, the advantage of this method is that there are enough $T_{m}$ values in the literature to enable a reasonable comparison for both saturated and unsaturated lipids. Table 2 shows the $R^{2}$ values for each of the parameter sets, Figure 2A shows the best data fits for changes in unsaturation and fatty acid distribution and Figure 2B shows the best data fit for changes in headgroup, which both stem from proxy T2-14, shown in Table 2.

The $\mathrm{R}^{2}$ values for Figure $2 \mathrm{~A}$ and Figure $2 \mathrm{~B}$ are 0.97 and 0.93 respectively, we repeated this analysis for the $\mathrm{C}_{20} \mathrm{PC}$ analogues shown in Table 1, which have more unsaturation than the C18 analogues, and found that parameter set T2-14 once again gave the highest $R^{2}$ score $\left(R^{2}\right.$ $=0.98)$, Table 2 . Since it is well established that the ordering effect of cholesterol is less significant for polyunsaturated lipids we anticipate that this method of refinement would underestimate the effect of cholesterol on the saturated and monounsaturated lipids present. Therefore to address the possible limitations of using parameter set T2-14 we considered how well it could account for the ordering effect of cholesterol on lipids containing saturated and monounsaturated fatty acid chains. This was accomplished using some of the limited amount of suitable order parameter data in the literature and assumes that the majority of the cholesterol associated with cell membranes is heterogeneous as discussed.

The orientational membrane order parameter for the lipids DOPC, SOPC and DPPC obtained in $10 \%$ cholesterol (Pan et al., 2008), are $0.32,0.40$ and 0.57 respectively. The value of $10 \%$ cholesterol was chosen because in membranes containing $L_{o}$ and $L_{d}$ phases of DOPC, DPPC and SOPC and cholesterol around $10 \%$ of the cholesterol was found to partition 
into the $L_{d}$ phase (Chen et al., 2007). In the absence of data for distearoylphosphatidylcholine (DSPC) we assume that the orientational order parameter of DPPC is identical to DSPC, within error. Since the theoretical maximum of the order parameter is 1 , then bearing in mind the inverse relationship between the order parameter and the output of the control function, plots of $w$ versus the order parameter of DOPC, DPPC and SOPC should go through 1. Confining the data in this way gives an $\mathrm{R}^{2}$ value of 0.96 for parameter set T2-14. Thus it would appear, within the limits of our coarse grain model, that parameter T2-14 is suited to model the ordering effects of cholesterol on lipids containing saturated and monounsaturated fatty acids and thus we use this parameter set to further analyse the data.

\subsection{Deconstructing lipid contributions to HVA inspired ratio control functions}

The values of $p_{\text {dis }}$ that emerge from parameter set T2-14 for each of the cell lines we have studied are $0.98 \pm 0.08,0.71 \pm 0.07$ and $0.46 \pm 0.01$ for $p_{\text {dis HL60, }} p_{\text {dis oleate }}$ and $p_{\text {dis HeLa }}$ respectively. Considering the HL60 and HeLa cell lines first, the fact that $p_{\text {dis HL6O }}$ and $p_{\text {dis HeLa }}$ are different suggests the disordered domains of these cell membranes have different optimal order. This is qualitatively consistent with fluorescence polarisation studies using DPH which have shown that the polarisation of the membranes of HL60 cells is 0.248 (Van Blitterswijk et al., 1987), whilst other studies at the same temperature and also with DPH show the polarisation of the membrane of HeLa cells is 0.232 (Fox and Delohery, 1987). An increase in polarisation indicates an increase in membrane disorder, so the values of $P_{\text {dis }}$ that emerge from our proxy are qualitatively consistent with these experimental observations. If we now consider HL60 cells in the presence and absence of exogenous oleate, we would expect the values of $p_{\text {dis HL6O }}$ and $p_{\text {dis oleate }}$ to be similar, on the assumption that membrane order is kept within tight boundaries, and this is what we see.

As a further measure we investigated whether or not any of the top ten parameter sets with the smallest standard deviation as shown in Figure 1 had $\mathrm{R}^{2}$ values comparable or greater than proxy T2-14, none were found. In addition we have explored ways of calibrating our proxy for membrane order to the microviscosity of a cell membrane, which is in the region of 100 to 300 centipoise (Wu et al., 2013). In principle this ought to be possible 
by calculating the $p_{\text {dis }}$ values, with parameter set $\mathrm{T} 2-14$, of mixed membrane bilayers with known viscosity. However there are insufficient microviscosity measurements of known composition mixed membranes to enable us to calibrate to. We can, however, use parameter set T2-14 to systematically understand how individual lipids contribute to increases and decreases in membrane order.

Figure 3 shows a plot of the individual $w$ values which emerge from parameter set T2-14 for each of the commonly occurring lipids in biological systems. The $w$ value of the pivot species PS 0:4 $\left(L_{p}\right)$ for parameter set T2-14 is 0.7544, which means that lipids with $w$ greater than 0.7544 contribute to increasing membrane disorder (relative to the pivot species) and lipids with lower values increase membrane order. There are no reported $T_{m}$ values for PS 0:4 lipids, such as PS 18:0/20:4c $\Delta^{5,8,11,14}$, however we can plot the individual $w$ values for each combination of headgroup and fatty acid using parameter set T2-14 to find lipid species with known $T_{m}$ values that have a $w$ value close to 0.7544 . This plot, Figure 3 , shows that, 0.7544 occurs somewhere between the $w$ value for PC 0:1 and PC 0:2.

Shown in Table 1, PC 18:0/18:1c $\Delta^{9}$ has a $T_{m}$ of $5.6^{\circ} \mathrm{C}$ and PC $18: 0 / 18: 2 c \Delta^{9,12}$ has a $T_{m}$ of $-16.5^{\circ} \mathrm{C}$, taking the midpoint between these two lipid species suggests that broadly speaking lipids with $T_{m}$ values less than $-5^{\circ} \mathrm{C}$ increase membrane disorder and lipids with $T_{m}$ values above $-5^{\circ} \mathrm{C}$ increase membrane order relative to the pivot species. The $T_{m}$ of PC 16:0/18:1c $\Delta^{9}$ is $-4{ }^{\circ} \mathrm{C}$ (Marsh, 2013; Tada et al., 2009), which is the closest value to that predicted by the parameter set T2-14. It is significant that the most dominant lipid in the HeLa and HL60 lipidomes is PC 16:0/18:1c $\Delta^{9}$ suggesting that all the lipids in the lipidome pivot around this species, at least under the $37^{\circ} \mathrm{C}$ cell culture conditions. It is also the second most abundant lipid in the HL60 oleate lipidome (Dymond et al., 2013) only slightly lower in abundance than $\mathrm{PC} 18: 1 c \Delta^{9} / 18: 1 c \Delta^{9}$. In a sense these observations are not surprising since we would expect the most dominant lipid species to exert the greatest effects on membrane order, however that this emerges from our data driven modelling is evidence of the robustness of the technique.

We can further explore the mechanisms by which the different cell types modify membrane order by looking at the individual contributions of lipids $\left(w_{n}\left[L_{d i s, n}\right]\right.$ or $w_{m}{ }^{-1}\left[L_{\text {ord }}\right.$, $m])$ to either the total numerator $\sum_{n=0}^{b} w_{n}\left[L_{d i s, n}\right]$ or total denominator $\sum_{m=0}^{a} \frac{1}{w_{m}}\left[L_{o r d, m}\right]$ of 
the control function (Equation 1). We are now at a distinct advantage since the control function in conjunction with parameter set T2-14 enables us to apply a consistent set of criteria to rationalise lipid compositional changes relative to the pivot species. Figure 4 ( $\mathrm{A}$ and $B$ ) shows the five most dominant lipids from the PC and PE headgroups for both the numerator and denominator of the control function using parameter set T2-14.

The dominant lipids in the PC contribution to the numerator are PC 16:0/18:1, PC 16:0/16:1, PC 16:0a/18:1, PC 16:0/ 16:0 and PC 18:0/18:1 in all three lipidomes, see Figure 4. The most dominant lipid is PC $16: 0 / 18: 1$, circa 15 to $20 \%$ of all the denominator species in all three lipidomes. A similar trend is seen in the PC species that contribute to the numerator with PC 18:1/18:1, PC 16:1/18:1, PC 18:1/18:2. PC 18:1/20:4 and PC 16:0/20:4 being the most dominant lipid species in the HL60 and HL60 oleate lipidomes. Significantly, the pivot species PC 16:0/ 18:1 splits the PC lipids into two sets i.e. the saturates and monounsaturates which contribute exclusively to the denominator (including PC16:0/18:1) and the polyunsaturates which contribute to the control function numerator.

Within the PE fraction there is more diversity in the five lipid species which contribute most to the numerator and denominator. PE 18:1/18:1 makes the biggest contribution to the numerator (from $10 \%$ to $30 \%$ ) in all three lipidomes. The other PE species PE 18:1/20:4, PE 18:0/22:6, PE18:0/22:5, PE 18:1/18:2 are scattered about the 5\% mark, with different lipidomes showing different trends. In the denominator the PE species show significant diversity, PE18:0/18:1 is the most dominating PE species in the HeLa and $\mathrm{HL60}$ oleate lipidomes and the second most dominating in the HL60 lipidome. The most dominating PE species in the HL60 lipidome is PE 18:0/20:4, which also features in the top 3 dominating lipids in the HeLa and $\mathrm{HL60}_{\text {oleate }}$ PE fractions. PE 16:0/18:1 and PE 18:0/20:2 also occur. In contrast to PC the denominator is contributed to by saturated, monounsaturated and polyunsaturated PE species. The numerator contains only polyunsaturated PE lipids. This latter observation illustrates the difficulty of judging the effects of individual lipid changes on membrane order, since increases in the polyunsaturated PE 18:0/ 20:4 might be expected to contribute to increasing membrane 
order. However our systematic approach and parameter set T2-14 show that PE 18:0/ 20:4 will in fact increase membrane order relative to the pivot lipid.

It is worth noting that in summation all the lipid species in Figure 4 make up in the region of 70 to $80 \%$ of the total lipid contribution to either the numerator or the denominator respectively, in all three lipidomes. In terms of their molar percentage, these lipid species are also the most dominant in all three lipidomes. The remaining 20 to $30 \%$ of the contribution to either the numerator or denominator of the control function is made up of by a combination of the remaining lipid species (circa 130).

It is interesting to consider the mechanism through which the HL6O cell line maintains membrane order in response to incubation with exogenous oleate. Figure 4 shows that in the PC contribution to the denominator, HL60 cells respond by elevating the PC 16:0/ 18:1 (from 13\% to 18\%) but at the loss of PC 16:0/ 16:1 (from 7\% to 4\%). In the numerator, Figure 4B, there is clear elevation in PC 18:1/ 18:1 (from $7 \%$ to $12 \%$ ) but this time at the expense of PC 16:1/ 18:1 (from 7\% to 3\%). In the PE fraction the contribution of PE 18:0/ 18:1 to the denominator increases from $13 \%$ to $18 \%$ in response to oleate, accompanied by a slight drop in PE 18:0/ 20:4, in the numerator there is a large increase in PE 18:1/ 18:1 (from 12\% to 30\%) accompanied by a small drop in the other 4 dominant species. Therefore in response to excess oleate which drives the production of more 18:1 containing lipid species, overall membrane order would appear to be maintained by subtle variance of the other dominant species. In particular it appears that 16:1 fatty acids are being replaced by 18:1 fatty acids in these dominant species.

If we consider the subcellular distribution of PC and PE lipids, found predominantly in the outer and inner membrane leaflets respectively, in conjunction with the results shown in Figure 4. Then given that the PC and PE lipid species are split across the both the denominator and numerator of the control function, it is plausible that the membrane order of each leaflet might be controlled independently. This is consistent with the observation of Seigneuret et al.(Seigneuret et al., 1984) who used ESR spin labelled analogues of PC, PS and PE lipids finding that the outer membrane leaflet of Human Erythrocytes was more ordered than the inner membrane leaflet. 


\subsection{Dynamics of the lipid contribution to membrane order in synchronised HeLa cells}

Previous work on adherent cells has shown that as Chinese Hamster Ovary (CHO) cells pass through the cell cycle, membrane 'fluidity', as measured by ESR using 5-doxylstearic acid as a spin probe, is at its highest during $\mathrm{G} 2 / \mathrm{M}$ and at its lowest in S phase with a low value observed in early G1 (Swartz et al., 1980). Since we have previously reported cell cycle changes (Hague et al., 2013) in the lipid content of adherent HeLa S3 cells we used parameter set T2-14 with these data. Figure 5 shows the variance of the $p_{\text {dis Hela sync }}$ using parameter set T2-14 over the cell cycle.

At 12 hours, when the majority of cells are in $\mathrm{G} 2 / \mathrm{M}$, the control function predicts a maximum in membrane disorder ( $p_{\text {dis Hela sync }} \approx 0.55$ ). Whilst at 9 hours when the majority of cells are in $S$ phase $p_{\text {dis Hela sync }} \approx 0.34$ and at a minimum, a slow decrease in $p_{\text {dis Hela sync }}$ is observed from the G1/ S boundary (0 hours). This is consistent with low 'fluidity' in S phase and high 'fluidity' in G2/M reported by Swartz et al. (Swartz et al., 1980)

To understand the origin of increased membrane disorder in $\mathrm{G} 2 / \mathrm{M}$ we looked at how the different lipid species change over the cell cycle. In the HeLa cell, total PC is maximal at 9 hours in S phase and total PE is maximal at 12 hours in G2/M (Hague et al., 2013). The temporal coincidence of high membrane disorder with high PE and low membrane disorder with high PC suggests that PE and PC lipids are driving the changes in $p_{\text {dis Hela sync }}$ seen in Figure 5. However since the PC and PE lipids are distributed in both the numerator and denominator of the control function, as shown in Figure 4, compositional changes in the PC and PE lipids must drive the fluctuations in the control function, Figure 5. To determine which of the lipid species are most dominant in bringing about the changes to membrane disorder observed in each of the phases of the cell cycle, we calculated the terms $w_{n}\left[L_{d i s, n}\right]$ or $w_{m}{ }^{-1}\left[L_{\text {ord, }}\right]$. For each lipid, these were expressed as a percentage of the total numerator $\sum_{n=0}^{b} w_{n}\left[L_{d i s, n}\right]$ or total denominator $\sum_{m=0}^{a} \frac{1}{w_{m}}\left[L_{o r d, m}\right]$ for each time point in the cell cycle lipidome of the HeLa cell, depending on which set the control function placed the lipid species into. Plots of the most dominant lipid species and strongest correlations with cell cycle phases are shown in supporting information (Figures S2 to S5) and briefly summarised below. 
PC 16:0/16:0 and PC 16:0/ 16:1 both contribute up to $7 \%$ of the denominator in late S phase (9 hours) from a $3 \%$ low in $\mathrm{G} 2 / \mathrm{M}$. The biggest single contributor to the denominator is PC 16:0/18:1 which peaks at $22 \%$ in late $S$ phase from a low of $15 \%$ in G2/M. PC 18:0/18:1 also contributes to the denominator peaking at $7 \%$ in late $S$ phase, dropping to $3 \%$ in G2/M. PC $16: 1 / 18: 1$ contributes to the numerator, peaking at $8 \%$ in late $S$ phase from around $5 \%$ in G2/M and G1. PC 18:1/ 18:1 follows the same trend peaking at $20 \%$ before dropping to $10 \%$ in $\mathrm{G} 2 / \mathrm{M}$.

In the denominator of the PE fraction, PE 16:0/18:1 falls through S phase from 4 to 2 \%, prior to rising back to $4 \%$ in $\mathrm{G} 2 / \mathrm{M}$ and then up to $6 \%$ in $\mathrm{G1}$. PE 18:0/18:1 is lowest in late S2, circa 9 hours on Figure S3, down to 6\% from about 10\%. PE 18:0/20:4 peaks at about $10 \%$ in $\mathrm{G} 2 / \mathrm{M}$, in $\mathrm{G} 1$ (at 15 hours) it drops to 3. In the numerator PE 18:1/ 18:1 drops during S phase down to $10 \%$ from $15 \%$, over the same time PE $18: 1 / 20: 4$ drops from $8 \%$ to $4 \%$. If we remove any of the above lipid species from the control function calculation we still see cell cycle dependent changes in $p_{\text {dis, }}$ this strongly suggests no single lipid species is driving the changes in membrane order observed.

All DAG, PS and PA species contributed less than $1 \%$ to either the numerator or denominator of the control function therefore we disregard these as having any specific role. In the PI species we see some correlations with the onset of G2/M (data not shown); however the changes observed are small in magnitude. If we remove all PI lipid species from the control function, the maximum at 12 hours, Figure 5, is still observed suggesting that these species are not significant in driving the membrane disorder seen at 12 hours. However, the coincidence of elevated PI species with the G2/M phase suggests they are associated with the PI-PLC cycle, cellular division (Sun et al., 1997) and nuclear signalling, detailed data driven modelling studies of the lipid composition of the nuclear envelope will be needed to further investigate this possibility.

\section{Conclusions}

Using a data driven modelling approach we have shown that a ratio control function for membrane disorder, inspired by HVA theory, is well evidenced within our lipidomic data 
sets, $c_{v(\text { mean })}$ in the range of 6 to $8 \%$. Refining of our parameter sets gave a parameter set (T2-14) with a $c_{v \text { (mean) }}$ of $6.93 \%$. This parameter set predicts that the cell membranes optimise disorder around the pivot lipid species $(w=0.7544)$ Lipids with $w$ values greater than this increase membrane disorder and lipids with $w$ values lower than this increase the optimised membrane order. With this parameter set we were able to systematically deconstruct the individual contribution of each of circa 130 lipid species to the control function and ultimately to membrane order. This implicated around 16 lipid species containing either a PC or PE headgroup, which had the biggest contribution to the control function. Parameter set T2-14 predicted the previously demonstrated (Swartz et al., 1980) high membrane 'fluidity' in the G2/M phase and low membrane 'fluidity' in S phase of the cell cycle. In synchronized populations of HeLa cells these same lipid species emerge as exerting the most effect on membrane order. We were unable to point to any single specific lipid species that might be used by the cell to drive global membrane disorder changes about the cell cycle.

One of the unanswered questions in our work is the connection between membrane disorder and the intrinsic curvature hypothesis. In particular our work raises the possibility that both membrane disorder and intrinsic curvature are separately regulated in vivo and that the changes in total phospholipid composition induced in response to environmental change reflect both control functions. Equally, however it is not implausible that regulation of the membrane disorder control function might concomitantly retain the intrinsic curvature control function with tight boundary conditions, or vice versa. This is a complex issue outside of the scope of this publication however it does set the direction for our future work.

Aside from allowing us to look in detail at the possible mechanisms behind phospholipid homeostasis, data driven modelling has enabled us to develop a systematic, evidence based approach that aids the understanding of large lipidomic datasets. It is possible that the best parameter set we report here (T2-14) might be used in conjunction with Equations 1 and 2 as a proxy for looking at the mechanisms by which membrane order is maintained in vivo. Of course the nature of data driven modelling is that parameter sets can be refined as more and more data sets become available. It will be interesting to see how a less coarse-grained approach in tandem with lipidomics of subcellular organelles and 
the heterogeneous 'lipid raft' structure with membranes builds a better picture of the in vivo biological membrane. What is clear from this work is that the systematic approach that emerges from data driven modelling of lipidomic data has the potential to begin the deconstruction of complex biological membrane systems.

\section{Acknowledgements}

The author would like thank George. S. Attard for helpful discussions and Lisa O'Rourke for proofreading the manuscript. 


\section{References}

Anderson, R.L., Minton, K.W., Li, G.C., Hahn, G.M., 1981. Temperature-induced homeoviscous adaptation of chinese hamster ovary cells. Biochim. Biophys. Acta Biomembr. 641, 334-348. doi:10.1016/0005-2736(81)90490-9

Behan, M.K., Macdonald, A.G., Jones, G.R., Cossins, A.R., 1992. Homeoviscous adaptation under pressure: the pressure dependence of membrane order in brain myelin membranes of deep-sea fish. Biochim. Biophys. Acta - Biomembr. 1103, 317-323. doi:10.1016/0005-2736(92)90102-R

Behan-Martin, M.K., Jones, G.R., Bowler, K., Cossins, A.R., 1993. A near perfect temperature adaptation of bilayer order in vertebrate brain membranes. Biochim. Biophys. Acta Biomembr. 1151, 216-222. doi:10.1016/0005-2736(93)90106-A

Bernabé Bloj, A., Moreroa, R.D., Faríasa, R.N., Truccob, R.E., 1973. Membrane lipid fatty acids and regulation of membrane-bound enzymes. Allosteric behaviour of erythrocyte $\mathrm{Mg2+-ATPase}$, and acetylcholineasterase from rats fed different fat-supplemented diets. Biochim. Biophys. Acta - Biomembr. 311, 67-79.

Calder, P.C., Bond, J.A., Harvey, D.J., Gordon, S., Newsholme, E.A., 1990. Uptake and incorporation of saturated and unsaturated fatty acids into macrophage lipids and their effect upon macrophage adhesion and phagocytosis. Biochem. J. 269, 807-814.

Cevc, G., 1991. How membrane chain-melting phase-transition temperature is affected by the lipid chain asymmetry and degree of unsaturation: an effective chain-length model. Biochemistry 30, 7186-7193.

Chattopadhyay, M.K., Jagannadham, M. V, 2001. Maintenance of membrane fluidity in Antarctic bacteria. Polar Biol. 24, 386-388. doi:10.1007/s003000100232

Chen, L., Yu, Z., Quinn, P.J., 2007. The partition of cholesterol between ordered and fluid bilayers of phosphatidylcholine: A synchrotron X-ray diffraction study. Biochim. Biophys. Acta - Biomembr. 1768, 2873-2881. doi:10.1016/j.bbamem.2007.07.023

Cooper, B.S., Hammad, L.A., Montooth, K.L., 2014. Thermal adaptation of cellular membranes in natural populations of Drosophila melanogaster. Funct. Ecol. 28, 886894. doi:10.1111/1365-2435.12264

Cossins, A.R., Macdonald, A.G., 1986. Homeoviscous adaptation under pressure. III. The fatty acid composition of liver mitochondrial phospholipids of deep-sea fish. Biochim. Biophys. Acta - Biomembr. 860, 325-335. doi:10.1016/0005-2736(86)90529-8

Cossins, A.R., Macdonald, A.G., 1984. Homeoviscous theory under pressure. Biochim. Biophys. Acta - Biomembr. 776, 144-150. doi:10.1016/0005-2736(84)90260-8 
Cossins, A.R., Prosser, C.L., 1978. Evolutionary adaptation of membranes to temperature. Proc. Natl. Acad. Sci. U. S. A. 75, 2040-2043.

Crockett, E.L., Hazel, J.R., 1995. Cholesterol levels explain inverse compensation of membrane order in brush border but not homeoviscous adaptation in basolateral membranes from the intestinal epithelia of rainbow trout. J. Exp. Biol. 198, 1105-1113.

Davy De Virville, J., Cantrel, C., Bousquet, A.L., Hoffelt, M., Tenreiro, A.M., Vaz Pinto, V., Arrabaca, J.D., Caiveau, O., Moreau, F., Zachowski, A., 2002. Homeoviscous and functional adaptations of mitochondrial membranes to growth temperature in soybean seedlings. Plant, Cell Environ. 25, 1289-1297. doi:10.1046/j.1365-3040.2002.00901.x

Demchenko, A.P., Mély, Y., Duportail, G., Klymchenko, A.S., 2009. Monitoring biophysical properties of lipid membranes by environment-sensitive fluorescent probes. Biophys. J. 96, 3461-3470. doi:10.1016/j.bpj.2009.02.012

Dymond, M., Attard, G., Postle, A.D., 2008. Testing the hypothesis that amphiphilic antineoplastic lipid analogues act through reduction of membrane curvature elastic stress. J. R. Soc. Interface 5, 1371-1386.

Dymond, M.K., Attard, G.S., 2008. Cationic type I amphiphiles as modulators of membrane curvature elastic stress in vivo. Langmuir 24, 11743-11751. doi:10.1021/la8017612

Dymond, M.K., Hague, C. V, Postle, A.D., Attard, G.S., 2013. An in vivo ratio control mechanism for phospholipid homeostasis: evidence from lipidomic studies. J. R. Soc. Interface 10, 20120854. doi:10.1098/rsif.2012.0854

Fox, M.H., Delohery, T.M., 1987. Membrane fluidity measured by fluorescence polarization using an EPICS V cell sorter. Cytometry 8, 20-25. doi:10.1002/cyto.990080104

Gawrisch, K., 2005. The Dynamics of Membrane Lipids, in: Yeagle, P.L. (Ed.), The Structure of Biological Membranes. CRC Press, Florida, pp. 147-171.

Georgiadi, A., Kersten, S., 2012. Mechanisms of gene regulation by fatty acids. Adv. Nutr. 3, 127-134. doi:10.3945/an.111.001602

Gladyshev, M.I., Semenchenko, V.P., Dubovskaya, O.P., Fefilova, E.B., Makhutova, O.N., Buseva, Z.F., Sushchik, N.N., Razlutskij, V.I., Lepskaya, E. V., Baturina, M.A., Kalachova, G.S., Kononova, O.N., 2011. Effect of temperature on contents of essential highly unsaturated fatty acids in freshwater zooplankton. Limnol. - Ecol. Manag. Inl. Waters 41, 339-347. doi:10.1016/j.limno.2011.03.001

Gonzalez, A., Odjélé, A., Weber, J.-M., 2013. PCB-153 and temperature cause restructuring of goldfish membranes: Homeoviscous response to a chemical fluidiser. Aquat. Toxicol. 144-145, 11-18. doi:10.1016/j.aquatox.2013.09.018

Gruner, S.M., 1985. Intrinsic Curvature Hypothesis for Biomembrane Lipid Composition: A role for Nonbilayer Lipids. Proc. Natl. Acad. Sci. U. S. A. 82, 3665-3669. 
Guschina, I.A., Harwood, J.L., 2006. Mechanisms of temperature adaptation in poikilotherms. FEBS Lett. 580, 5477-5483. doi:10.1016/j.febslet.2006.06.066

Hague, C. V., Postle, A.D., Attard, G.S., Dymond, M.K., 2013. Cell cycle dependent changes in membrane stored curvature elastic energy: evidence from lipidomic studies. Faraday Discuss. 161, 481-497. doi:10.1039/c2fd20078c

Hazel, J.R., 1997. "Thermal Aaptation in Biological Membranes: Beyond Homeoviscous Adaptation." Adv. Mol. cell Biol. 19, 57-102.

Hazel, J.R., 1995. Thermal adaptation in biological membranes: is homeoviscous adaptation the explanation? Annu. Rev. Physiol. 57, 19-42. doi:10.1146/annurev.ph.57.030195.000315

Hermansson, M., Hokynar, K., Somerharju, P., 2011. Progress in Lipid Research Mechanisms of glycerophospholipid homeostasis in mammalian cells. Prog. Lipid Res. 50, 240-257. doi:10.1016/j.plipres.2011.02.004

Kaiser, H.-J., Lingwood, D., Levental, I., Sampaio, J.L., Kalvodova, L., Rajendran, L., Simons, K., 2009. Order of lipid phases in model and plasma membranes. Proc. Natl. Acad. Sci. U. S. A. $106,16645-16650$. doi:10.1073/pnas.0908987106

King, M.E., Spector, A.A., 1978. Effect of specific fatty acyl enrichments on membrane physical properties detected with a spin label probe . J. Biol. Chem. 253, 6493-6501.

Koynova, R., Caffrey, M., 1998. Phases and phase transitions of the phosphatidylcholines. Biochim. Biophys. Acta 1376, 91-145.

Larijani, B., Dufourc, E.J., 2006. Polyunsaturated phosphatidylinositol and diacylglycerol substantially modify the fluidity and polymorphism of biomembranes: a solid-state deuterium NMR study. Lipids 41, 925-932.

Laroche, C., Beney, L., Marechal, P.A., Gervais, P., 2001. The effect of osmotic pressure on the membrane fluidity of Saccharomyces cerevisiae at different physiological temperatures. Appl. Microbiol. Biotechnol. 56, 249-254. doi:10.1007/s002530000583

Marsh, D., 2013. Handbook of lipid bilayers. CRC Press.

Marsh, D., 1999. Thermodynamic analysis of chain-melting transition temperatures for monounsaturated phospholipid membranes: dependence on cis-monoenoic double bond position. Biophys. J. 77, 953-963. doi:10.1016/S0006-3495(99)76946-8

Mazzella, N., Syakti, a, Molinet, J., Gilewicz, M., Doumenq, P., Artaud, J., Bertrand, J., 2005. Effects of crude oil on phospholipid fatty acid compositions of marine hydrocarbon degraders: estimation of the bacterial membrane fluidity. Environ. Res. 97, 300-311. doi:10.1016/j.envres.2004.06.007 
Morrow, M.R., Whitehead, J.P., Lu, D., 1992. Chain-length dependence of lipid bilayer properties near the liquid crystal to gel phase transition. Biophys. J. 63, 18-27.

Oger, P.M., Cario, A., 2013. Adaptation of the membrane in Archaea. Biophys. Chem. 183, 42-56. doi:10.1016/j.bpc.2013.06.020

Ortiz, A., Villalaín, J., Gómez-Fernández, J.C., 1988. Interaction of diacylglycerols with phosphatidylcholine vesicles as studied by differential scanning calorimetry and fluorescence probe depolarization. Biochemistry 27, 9030-9036.

Ozawa, Y.N., 2011. Review Adaptive regulation of membrane lipids and fluidity during thermal acclimation in Tetrahymena. Proc. Jpn. Acad., Ser. B 87, 450-462.

Pan, J., Mills, T.T., Tristram-Nagle, S., Nagle, J.F., 2008. Cholesterol perturbs lipid bilayers nonuniversally. Phys. Rev. Lett. 100, 1-4. doi:10.1103/PhysRevLett.100.198103

Parsons, J.B., Rock, C.O., 2013. Bacterial lipids: metabolism and membrane homeostasis. Prog. Lipid Res. 52, 249-276. doi:10.1016/j.plipres.2013.02.002

Peng, A.A., Pisal, D.S., Doty, D., Balu-Lyer, S.C., 2012. Phosphatidylinositol induces fluid phase formation and packing defects in phosphatidylcholine model membranes. Chem Phys Lipids. 165, 15-22. doi:10.1016/j.chemphyslip.2011.10.002.Phosphatidylinositol

Rybczynska, M., Spitaler, M., Knebel, N.G., Boeck, G., Grunicke, H., Hofmann, J., 2001. Effects of miltefosine on various biochemical parameters in a panel of tumor cell lines with different sensitivities. Biochem. Pharmacol. 62, 765-772. doi:10.1016/S00062952(01)00715-8

Santoro, N., Lisi, A., Pozzi, D., Pasquali, E., Serafino, A., Grimaldi, S., 1997. Effect of extremely low frequency (ELF) magnetic field exposure on morphological and biophysical properties of human lymphoid cell line (Raji). Biochem. Biophys. Acta 1357, 281-290.

Seigneuret, M.P., Zachowski, A., Hermann, A., Devaux, P.F., 1984. Asymmetric lipid fluidity in human erythrocyte membrane: new spin-label evidence. Biochemistry 23, 4271-4275.

Simons, K., Ikonen, E., 2000. How Cells Handle Cholesterol. Science (80-. ). 290, 1721-1727.

Simons, K., Sampaio, J., 2011. Membrane organization and lipid rafts. Cold Spring Harb. Perspect. Biol. 3, 1-18.

Sinensky, M., 1974. Homeoviscous adaptation--a homeostatic process that regulates the viscosity of membrane lipids in Escherichia coli. Proc. Natl. Acad. Sci. U. S. A. 71, 522525.

Sun, B., Murray, N.R., Fields, A.P., 1997. A Role for Nuclear Phosphatidylinositol-specific Phospholipase C in the G2/M Phase Transition. J. Biol. Chem. 272, 26313-26317. doi:10.1074/jbc.272.42.26313 
Swartz, H.M., Lai, C.S., LE, H., 1980. Electron spin resonance studies of changes in membrane fluidity of Chinese hamster ovary cells during the cell cycle. Biochim Biophys Acta. 602, 117-126.

Tada, K., Miyazaki, E., Goto, M., Tamai, N., Matsuki, H., Kaneshina, S., 2009. Barotropic and thermotropic bilayer phase behavior of positional isomers of unsaturated mixed-chain phosphatidylcholines. Biochim. Biophys. Acta 1788, 1056-1063.

doi:10.1016/j.bbamem.2009.02.008

Van Blitterswijk, W.J., Hilkmann, H., Storme, G.A., 1987. Accumulation of an Alkyl Lysophospholipid in Tumor Cell Membranes Affects Membrane Fluidity and Tumor Cell Invasion. Lipids 11, 820-823.

Varga, T., Czimmerer, Z., Nagy, L., 2011. PPARs are a unique set of fatty acid regulated transcription factors controlling both lipid metabolism and inflammation. Biochim. Biophys. Acta 1812, 1007-1022. doi:10.1016/j.bbadis.2011.02.014

Vigh, L., Maresca, B., Harwood, J.L., 1998. Does the membrane's physical state control the expression of heat shock and other genes? Trends Biochem. Sci. 10, 369-374.

Wade, H.L., 2004. In basic and advanced regulatory control: system design and application. Triangle Park, NC: ISA.

Wassall, S.R., Brzustowicz, M.R., Shaikh, S.R., Cherezov, V., Caffrey, M., Stillwell, W., 2004. Order from disorder, corralling cholesterol with chaotic lipids: The role of polyunsaturated lipids in membrane raft formation. Chem. Phys. Lipids 132, 79-88. doi:10.1016/j.chemphyslip.2004.09.007

Wu, Y., Stefl, M., Olzyńska, A., Hof, M., Yahioglu, G., Yip, P., Casey, D.R., Ces, O., Humpolíčková, J., Kuimova, M.K., 2013. Molecular rheometry: direct determination of viscosity in Lo and Ld lipid phases via fluorescence lifetime imaging. Phys. Chem. Chem. Phys. 15, 14986-14993. doi:10.1039/c3cp51953h

Xu, Y., Heath, R.J., Li, Z., Rock, C.O., White, S.W., 2001. The FadR.DNA complex. Transcriptional control of fatty acid metabolism in Escherichia coli. J. Biol. Chem. 276, 17373-17379. doi:10.1074/jbc.M100195200

Yu, Y., Vidalino, L., Anesi, A., Macchi, P., Guella, G., 2014. A lipidomics investigation of the induced hypoxia stress on HeLa cells by using MS and NMR techniques. Mol. Biosyst. 10, 878-890. doi:10.1039/c3mb70540d 


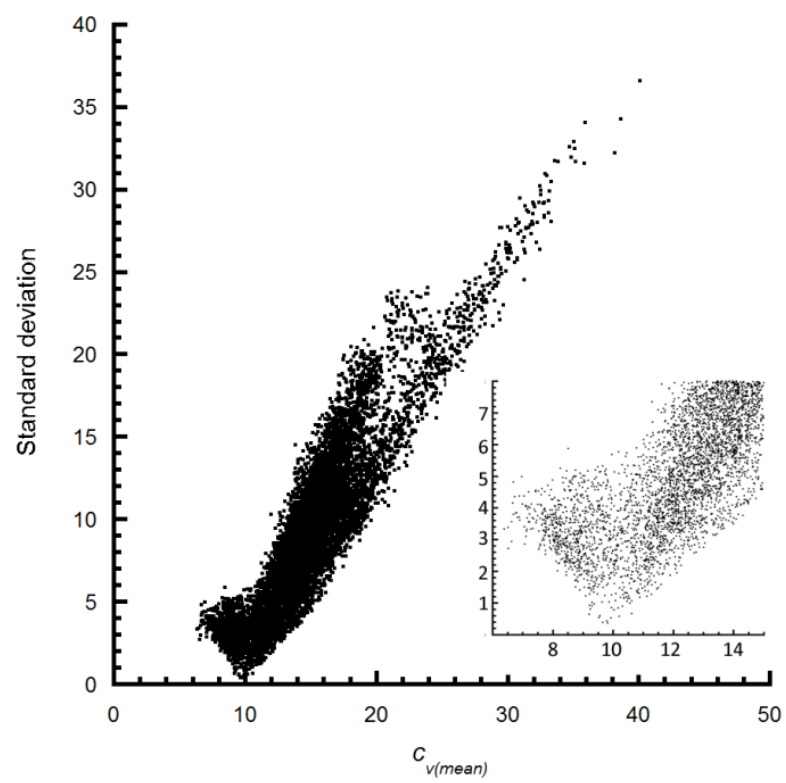

Figure $1 c_{v \text { mean }}$ versus standard deviation for data derived from a ratio control function (Equation 1) for membrane disorder. Values towards the bottom left of the graph, expanded in the inset, show parameter sets that give consistently the lowest variance in $p_{\text {dis }}$ across all cell types. The 15 parameter sets giving the lowest $c_{v \text { mean }}$ values are shown in Table 2.
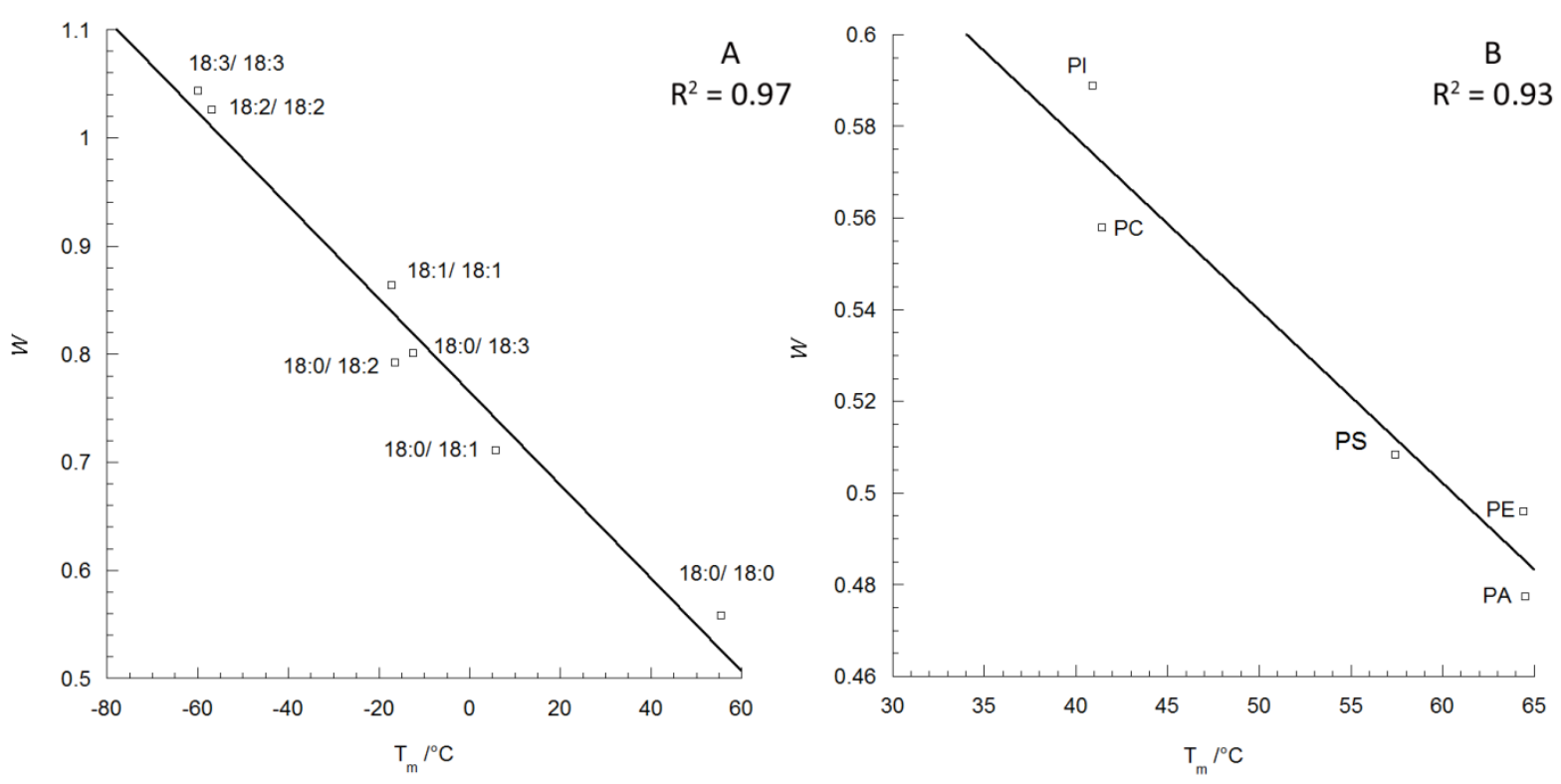

Figure 2, linear fits of $T_{m}$ versus $w$ for a series of biologically relevant $\mathrm{C}_{18} \mathrm{PC}$ lipids with different unsaturation distributions $(A)$ and dipalmitoyl lipids with different headgroups (B), $w$ values are derived from Equation 2 and parameter set T2-14, full lipid structural details and $T_{m}$ values are given in Table 1. 


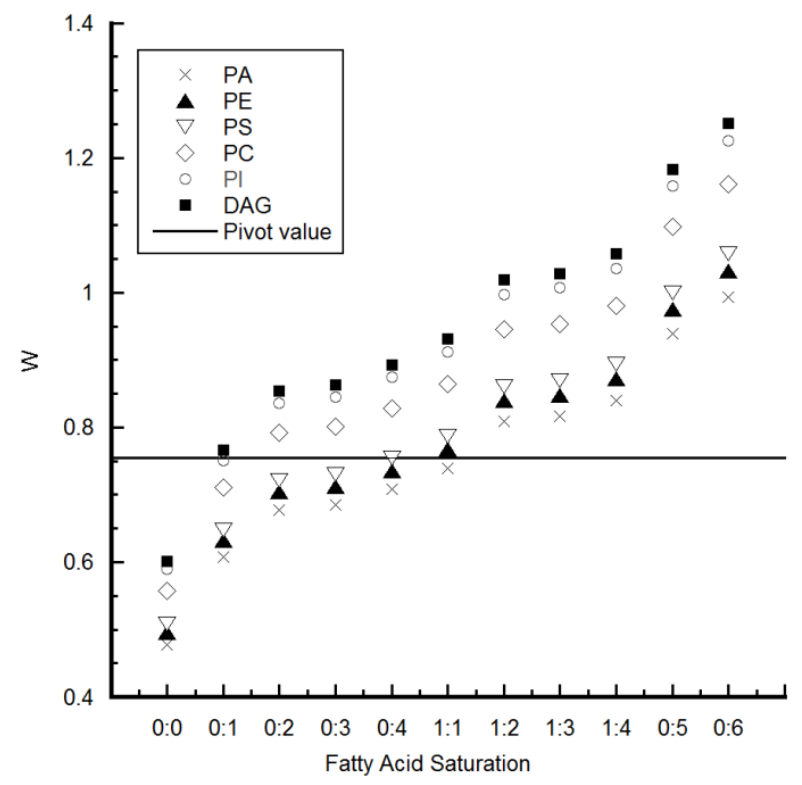

Figure 3, the distribution of the major lipids found in mammalian cells as ranked by the $w$ values that emerge from parameter set T2-14. The bold line shows the $w$ value of the pivot species, lipids above the pivot contribute to the control function numerator $\left(L_{\text {dis }}\right)$ and net membrane disorder. Lipids below the pivot lipid, and including the pivot species contribute to the denominator ( $\left.L_{\text {ord }}\right)$ of the control function and net membrane order. 

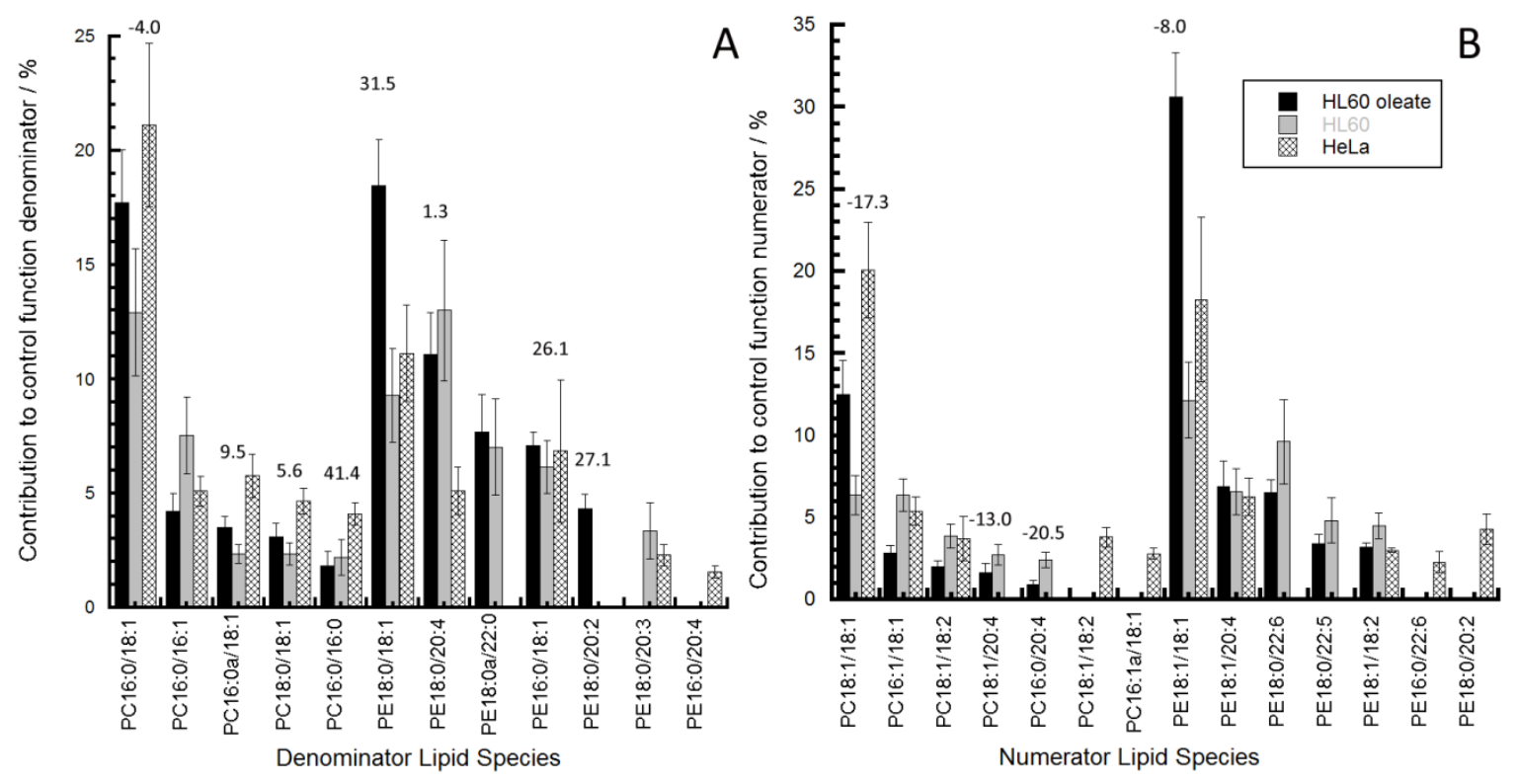

Figure 4, $(A$ and $B)$ the percentage contribution of the five most dominant $P C$ and $P E$ lipid species to the denominator and numerator of the control function shown in Equation 1. Values were obtained using parameter set T2-14, where available in the literature (Marsh, 2013) $T_{m} /{ }^{\circ} \mathrm{C}$ values are shown on the figure. 


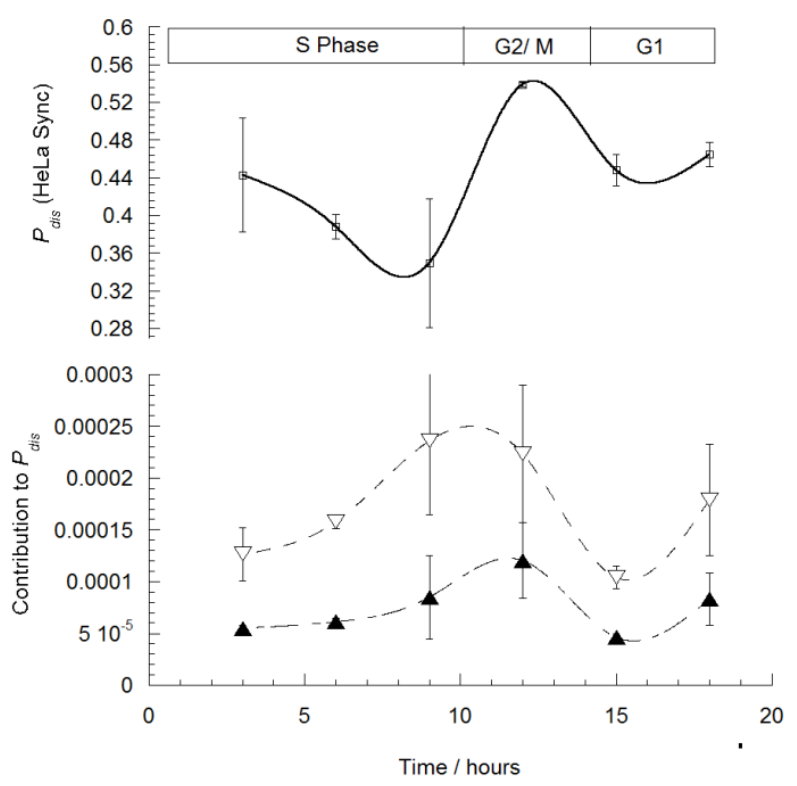

Figure 5 variance predicted by parameter set T2-14 using cell cycle associated lipid compositional changes (empty squares), sum of the species that contribute to the control function denominator $\sum_{m=0}^{a} \frac{1}{w_{m}}\left[L_{o r d, m}\right]$ (empty inverted triangles) and sum of the species that contribute to the control function numerator $\sum_{n=0}^{b} w_{n}\left[L_{d i s, n}\right]$ (filled triangles). Lipid compositions and cell cycle data were obtained from Hague et al. (Hague et al., 2013). 
Table $1 T_{m}$ values for biologically occurring lipid isomers obtained from Marsh (Marsh, 2013) and used to rank the coarse grained contributions to membrane disorder within control functions shown in Equations 1 and 2.

\begin{tabular}{cc} 
Lipid & $\boldsymbol{T}_{m} /{ }^{\circ} \mathrm{C}$ \\
\hline PC 18:0/18:0 & 55.3 \\
PC 18:0/18:1c $\Delta^{9}$ & 5.6 \\
PC 18:0/18:2c $\Delta^{9,12}$ & -16.5 \\
PC 18:0/18:3c $\Delta^{9,12,15}$ & -12.5 \\
PC 18:1c $\Delta^{9} / 18: 1 c \Delta^{9}$ & -17.3 \\
PC 18:2c $\Delta^{9,12} / 18: 2 c \Delta^{9,12}$ & -57 \\
PC 18:3c $\Delta^{9,12,15} / 18: 3 c \Delta^{9,12,15}$ & -60 \\
& \\
PC 20:0/20:0 & 66.4 \\
PC 20:0/20:1c $\Delta^{11}$ & 19.7 \\
PC 20:0/20:2c $\Delta^{11,14}$ & 1.8 \\
PC 20:0/20:3c ${ }^{11,14,17}$ & 4.4 \\
PC 20:0/20:4c $\Delta^{5,8,11,14}$ & -6.8 \\
PC 20:1c $\Delta^{11} / 20: 1 c \Delta^{11}$ & -4.3 \\
PC 20:4c $\Delta^{5,8,11,14} / 20: 4 c \Delta^{5,8,11,14}$ & -69.2 \\
PA 16:0/16:0 & \\
PE 16:0/16:0 & 64.5 \\
PS 16:0/16:0 & 64.4 \\
PC 16:0/16:0 & 57.4 \\
PI 16:0/16:0 & 41.4 \\
\hline
\end{tabular}


Table 2, the fifteen parameter sets that gave the least variance across ratio control functions derived from lipid contributions to membrane order.

$\mathrm{R}^{2}$ values are for $\mathrm{C} 18, \mathrm{C} 20$ and headgroup analogues shown in Table 1.

\begin{tabular}{|c|c|c|c|c|c|c|c|c|c|c|c|c|c|c|c|}
\hline $\boldsymbol{P}_{\text {dis identifier }}$ & $\mathrm{T} 2-1$ & $\mathrm{~T} 2-2$ & $\mathrm{~T} 2-3$ & $\mathrm{~T} 2-4$ & $\mathrm{~T} 2-5$ & $\mathrm{~T} 2-6$ & $\mathrm{~T} 2-7$ & $\mathrm{~T} 2-8$ & T2-9 & $\mathrm{T} 2-10$ & $\mathrm{~T} 2-11$ & $\mathrm{~T} 2-12$ & $\mathrm{~T} 2-13$ & T2-14 & T2-15 \\
\hline$C_{h 1} 0$ & 0.11 & 0.18 & 0.06 & 0.1 & 0.12 & 0.14 & 0.07 & 0.13 & 0.13 & 0.09 & 0.06 & 0.23 & 0.04 & 0.31 & 0.03 \\
\hline$c_{h 1} 1$ & 0.34 & 0.43 & 0.29 & 0.45 & 0.36 & 0.35 & 0.14 & 0.42 & 0.27 & 0.25 & 0.35 & 0.39 & 0.2 & 0.48 & 0.18 \\
\hline$c_{h 1} 2$ & 0.35 & 0.49 & 0.3 & 0.53 & 0.39 & 0.45 & 0.25 & 0.43 & 0.37 & 0.33 & 0.36 & 0.53 & 0.37 & 0.57 & 0.2 \\
\hline$C_{h 13}$ & 0.36 & 0.54 & 0.4 & 0.54 & 0.47 & 0.56 & 0.25 & 0.45 & 0.37 & 0.4 & 0.45 & 0.72 & 0.4 & 0.58 & 0.25 \\
\hline$c_{h 1} 4$ & 0.65 & 0.78 & 0.42 & 0.81 & 0.61 & 0.65 & 0.26 & 0.78 & 0.58 & 0.48 & 0.54 & 0.76 & 0.48 & 0.61 & 0.4 \\
\hline$c_{h 1} 5$ & 0.72 & 0.84 & 0.52 & 0.83 & 0.74 & 0.73 & 0.64 & 0.84 & 0.65 & 0.78 & 0.77 & 0.82 & 0.59 & 0.91 & 0.76 \\
\hline$c_{h 1} 6$ & 0.85 & 0.91 & 0.97 & 0.91 & 0.83 & 0.8 & 0.8 & 0.87 & 0.75 & 0.9 & 0.95 & 0.86 & 0.83 & 0.98 & 0.83 \\
\hline$H_{g P A}$ & 0.53 & 0.57 & 0.58 & 0.63 & 0.62 & 0.36 & 0.29 & 0.45 & 0.63 & 0.66 & 0.56 & 0.77 & 0.35 & 0.77 & 0.27 \\
\hline$H_{g}$ PE & 0.7 & 0.81 & 0.72 & 0.74 & 0.83 & 0.41 & 0.65 & 0.51 & 0.76 & 0.76 & 0.68 & 0.85 & 0.55 & 0.8 & 0.44 \\
\hline$H_{g P S}$ & 0.73 & 0.81 & 0.74 & 0.78 & 0.85 & 0.44 & 0.68 & 0.51 & 0.81 & 0.94 & 0.75 & 0.9 & 0.62 & 0.82 & 0.45 \\
\hline$H_{g P C}$ & 0.78 & 0.9 & 0.74 & 0.87 & 0.85 & 0.48 & 0.69 & 0.55 & 0.92 & 0.95 & 0.76 & 0.91 & 0.62 & 0.9 & 0.48 \\
\hline$H_{g P I}$ & 0.9 & 0.93 & 0.81 & 0.9 & 0.91 & 0.48 & 0.79 & 0.7 & 0.93 & 0.95 & 0.81 & 0.98 & 0.65 & 0.95 & 0.53 \\
\hline$H_{g D A G}$ & 0.99 & 0.98 & 0.96 & 0.93 & 0.93 & 0.49 & 0.85 & 0.76 & 0.93 & 0.98 & 0.97 & 0.99 & 0.82 & 0.97 & 0.56 \\
\hline$L_{p(\text { species })}$ & DAG 0:3 & PI 1:2 & PS 0:4 & DAG 0:2 & PA 0:2 & PA 0:3 & DAG 0:1 & DAG 0:2 & DAG 0:1 & DAG 0:1 & DAG 0:1 & DAG 0:1 & DAG 0:1 & PS 0:4 & PS 0:3 \\
\hline$L_{p(v a l u e)}$ & 0.4653 & 0.6696 & 0.3552 & 0.5859 & 0.4464 & 0.2520 & 0.1785 & 0.4256 & 0.3720 & 0.3332 & 0.3977 & 0.6138 & 0.1968 & 0.7544 & 0.126 \\
\hline$c_{v(\text { mean })}$ & 6.44 & 6.55 & 6.60 & 6.65 & 6.69 & 6.70 & 6.71 & 6.71 & 6.79 & 6.83 & 6.85 & 6.92 & 6.93 & 6.93 & 7.00 \\
\hline s.d. & 3.30 & 3.35 & 2.69 & 3.55 & 4.14 & 3.78 & 4.80 & 2.90 & 3.82 & 3.69 & 3.83 & 3.68 & 4.35 & 4.43 & 4.12 \\
\hline$R^{2}(C 18)$ & 0.86 & 0.94 & 0.86 & 0.93 & 0.90 & 0.91 & 0.92 & 0.86 & 0.97 & 0.92 & 0.87 & 0.79 & 0.94 & 0.97 & 0.90 \\
\hline$R^{2}(C 20)$ & 0.67 & 0.87 & 0.93 & 0.86 & 0.92 & 0.96 & 0.94 & 0.71 & 0.82 & 0.96 & 0.91 & 0.89 & 0.96 & 0.98 & 0.85 \\
\hline$R^{2}(\mathrm{Hg})$ & 0.69 & 0.61 & 0.50 & 0.84 & 0.43 & 0.83 & 0.45 & 0.64 & 0.84 & 0.67 & 0.65 & 0.70 & 0.49 & 0.93 & 0.55 \\
\hline
\end{tabular}

